\title{
Roster of Members
}

\author{
PATRON \\ University of California \\ SUSTAINING
}

Henry E. Abt, President, Brand Names Foundation, 37 W. 57th Street, New York 19, N.Y.

Charles M. Goethe, Capital National Bank Building, Sacramento 14, California

Erwin G. Gudde, P. O. Box 512, Orinda, California

James K. Moffitt, Piedmont 11, California

Elsdon C. Smith, 322 Sherman Avenue, Evanston, Illinois

Walter Yust, Editor, Encyclopaedia Britannica, Chicago 11, Illinois

\section{ACTIVE}

Samuel H. Abramson, 294 Fisher Ave., White Plains, N.Y. (Jewish and French Canadian names in U. S.)

Louise M. Ackerman, Box 210, RFD 1, Lincoln, Neb.

R. W. Ackerman, Dept. of English, Stanford Univ., Stanford, Calif.

K. T. Adams, $5^{110}$ Mesa Terrace, La Mesa, Calif.

Curtis Adler, 815 East 14th St., Brooklyn 30, N.Y. (Family names)

Dr. D. T. Aitken, Parkside Hospital, Macclesfield, Cheshire, England

Edwin C. Alberts, Box 1061, Estes Park, Colorado, (Western U.S. place names)

Gerard Lord Alexander, Map Division, Public Library, New York 18, N.Y. (Nicknames of American cities; American personal names)

Harold B. Allen, 317 Folwell Hall, Univ. of Minn., Minneopolis 14, Minn. (Linguistic atlas, upper Midwest)

Morse S. Allen, 3 Fern St., Hartford 5, Conn. (Connecticut place names)

Karl Ames, $3^{8} 3$ Oak Ave., River Edge, N.J.

William E. Ashton, 712 Fifth Avenue, Helena, Mont. (Postoffice and county names)

Donald Gay Baker, 8th Ave., Collegeville, Penn.

Clarence L. Barnhart, 141 Parkway Rd., Bronxville 8, N.Y. (Lexicography)

Mary Eileen Barry, Southern Ill. University, Carbondale, Ill.

A. H. Batchelder, 833 Mendocino Ave., Berkeley 7, Calif.

Madison S. Beeler, 2413 Dwinelle Hall, Berkeley 4, Calif., (Indo-European names)

Allen Belden, 7055 Wyndale Street, N.W., Washington 15, D.C.

Handsel G. Bell, 1605 Fairview St., Berkeley 3, Calif.

A. I. Bereskin, Dept. of Natural Resources, Regina, Sask., Canada

Roy M. Bickford, 2339 Torry Road, Route 6, Flint, Mich.

A. Boodberg, 105 Durant Hall, Berkeley 4, Calif. (Far Eastern names)

F. W. Bradley, $425^{\circ}$ St. Clair Dr., Columbia 4, S.C. (South Carolina place names)

E. L. Brainerd, 2814 Polk St., N.E., Minneapolis 18, Minn.

A. A. Brierly, Independence, Calif. 
Charles Vyner Brooke, $425^{6}$ West $15^{\text {th }}$ Ave., Vancouver 8, B.C., Canada

Lucille C. Brouillet, G. \& C. Merriam Co., 47 Federal St., Springfield 2, Mass.

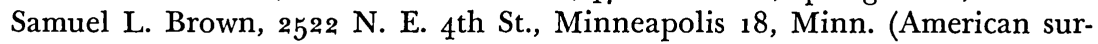
names of European origin)

Thomas P. Brown, 615 Alvarado St., San Francisco 14, Calif. (Calif. and western names)

Margaret M. Bryant, I Montague Terrace, Brooklyn 2, N.Y.

Warren H. Buell, 608 North Cherokee Ave., Los Angeles 4, Calif.

Meredith F. Burrill, U.S. Board on Geographic Names, Washington, D.C.

Arthur L. Campbell, 5066 Romaini St., Los Angeles 29, Calif. (American family names)

A. Carnoy, University of Louvain, Belgium

Frederic G. Cassidy, $3{ }_{15}$ Bascom Hall, Madison 6, Wis.

Bennett A. Cerf, President, Random House, Inc., New York 22, N.Y.

Ben F. Cheek, Jr., P.O. Box 387 , Toccoa, Georgia

Lewis H. Chrisman, 87 Smithfield St., Buckhannon, West Virginia

Marjorie Clagett, Western State College, Bowling Green, Kentucky (French names in Kentucky. Biological names)

Donald T. Clark, 25 Moon Hill Rd., Lexington, Mass. (Place names, Trade names)

Dwight L. Clarke, 434 Muirfield Road, Los Angeles 5, Calif.

R. T. Cochran, 1047 Simmons St., Kirkwood 22, Missouri

D. B. Cofer, Box 211, Faculty Exchange, College Station, Texas (Meaning of surnames)

Hennig Cohen, Univ. of South Carolina, Columbia, S.C.

Henry E. Collins, 823 La Porte Ave., Valparaiso, Indiana

Charles W. Collins, 716 Brummel St., Evanston, Ill.

Franklin D. Cooley, Univ. of Maryland, College Park, Md.

Joseph N. Corcoran, 1304 Lincoln-Library Bldg., Philadelphia 7, Penn.

E. H. Criswell, Dept. of English, Univ. of Tulsa, Tulsa 4, Okla.

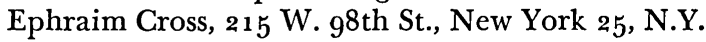

Thomas L. Crowell, 15 Claremont Ave., New York 27, N.Y.

Jack A. Dabbs, 1011 Edgewood, Bryant, Texas (Latin American toponymy)

Levette J. Davidson, University of Denver, Denver 10, Colo. (Colorado names)

J. Frank Dawson, 628 Patterson Bldg., Denver 2, Colo. (Colorado place names)

Robert N. De Armond, Box 1571, Juneau, Alaska (Alaska place names)

Henri Draye, Herent, Louvain, Belgium

Geart R. Droege, Dept. of Geography, Louisiana State Univ., Baton Rouge 3, La. (Frisian names)

Aubrey Drury, 1540 Euclid Ave., Berkeley 8, Calif. (Personal and place names) Audrey R. Duckert, G. \& C. Merriam Co., 47 Federal St., Springfield, Mass. (Place names of Massachusetts)

Louis Blake Duff, 18 King St., Welland, Ontario, Canada (North American names)

R. M. Duncan, Univ. of New Mexico, Albuquerque, New Mexico

A. R. Dunlap, 320 Hullihen Hall, Newark, Del.

E. C. Ehrensperger, Univ. of South Dakota, Vermillion, South Dakota (South Dakota place names)

Karl M. Elish, Middleburgh, N.Y.

Bert Emsley, 205 Derby Hall, Ohio State Univ., Columbus 10, Ohio

E. E. Ericson, Gustavus Adolphus College, St. Peter, Minn. 
Francis P. Farquhar, 2930 Avalon Ave., Berkeley 5, Calif. (Sierra Nevada names)

Olof von Feilitzen, Kungl. Biblioteket, Stockholm 5, Sweden

Neil Green Findly, Box 217 , Canyon, Texas

Theodore G. Foster, 2426 Strathmore Rd., Lansing 10, Mich. (Michigan place names)

Goesta Franzen, Faculty Exchange, Univ. of Chicago, Chicago 37, Ill. (Scandinavian names)

Edward A. H. Fuchs, 39 Clifton Ave., Springfield 5, Mass. (Lexicography)

Joseph G. Fucilla, Northwestern University, Evanston, Ill. (Italian personal names)

E. Trible Gatewood, Professional Bldg., Richmond 19, Va. (Personal and scientific names)

James A. Geary, 4306 28th Place, Mt. Rainier, Md. (Indo-European, esp. Celtic names. Algonquian names)

Mrs. Davis E. Geiger, $5^{\circ 9}$ Amanda Furnace Dr., Ashland, Ky. (Surnames and place names)

I. J. Gelb, Univ. of Chicago, Chicago, Ill.

Demetrius J. Georgacas, Univ. of North Dakota, Grand Forks, North Dakota

Martha Jane Gibson, Talladega College, Talladega, Ala. (Place names, surnames, trade names)

Deniston Gilbert, P. O. Box 8816, Univ. Station, Baton Rouge 4, La.

William H. Gilbert, 9015 First Ave., Silver Spring, Md. (Relation of U.S. surnames to names of clans and gentile groups)

Chas. Edgar Gilliam, 220 Union Trust Bldg., Petersburg, Va. (Folk names)

Ben Grauer, 1 West 67 th St., New York 23, N.Y. (Etymology of names)

W. Cabell Greet, Barnard College, New York 27, N.Y.

Ewald T. Grether, School of Business Administration, Univ. of Calif., Berkeley 4, Calif. (Trade names)

Mayme L. Hamlett, Southwest Baptist College, Bolivar, Missouri

Stratton O. Hammon, 1104 Washington Blvd., Louisville 2, Kentucky (Surnames 5 ooo B.C. to the present)

John Louis Haney, 6419 Woodpine Ave., Philadelphia 31, Penn.

Angella M. Hanley, 371 Falconer St., N. Tonawanda, N.Y.

Phil Townsend Hanna, Terminal Annex, Box 289o, Los Angeles 54, Calif. (California land names)

Lester Hargreth, 2106 Spence St., Tallahassee, Florida

John Harrington, Smithsonian Institution, Washington, D.C. (Indian names)

Jesse W. Harris, Southern Illinois University, Carbondale, Ill.

James D. Hart, 740 San Luis Rd., Berkeley 7, Calif.

Lee F. Hawley, 1 Langdon St., Madison 3, Wis. (Place and family names in western New York state)

David Judson Haykin, Library of Congress, Washington 25, D.C.

Charles Haywood, Queens College, Flushing 67, N.Y. (North American place name bibliography. Long Island names)

Lewis Heck, 3421 Northampton St., Washington 15, D.C. (Coast and Geodetic Survey)

Atcheson L. Hench, Univ. of Virginia, Charlottesville, Va.

Max J. Herzberg, 135 Mercer Place, South Orange, New Jersey

Archibald A. Hill, 1719 Mass Ave., N.W., Washington 6, D.C.

Robert Hitchman, 6111 gth Ave. N., Seattle 2, Wash. 
F. W. Hodge, Southwest Museum, Highland Park, Los Engeles 42, Calif. (Indian names)

Cecilia A. Hotchner, Hunter College, 695 Park Ave., New York 21, N.Y.

Arthur H. Hughes, Trinity College, Hartford 6, Conn. (Connecticut place names)

Walter Irving, 3 Mather St., Binghamton, New York (Indian names. Local history in place names)

Wyatt Jacobs, 221 N. La Salle St., Chicago, Ill.

Assar Janzén, Univ. of Calif., Berkeley 4, Calif. (Scandinavian names)

Philip N. Jenner, DAC, HQ 5ooth Mil Intel Svc GP, APO 613 \% PM, San Francisco, Calif.

Lloyd B. Jensen, 7300 South Shore Dr., Chicago 49, Ill. (Norman names)

E. Gustav Johnson, 3225 Foster Ave., Chicago 25, Ill.

Petras Jonikas, 281 Olmstead Road, Riverside, Ill. (Lithuanian names)

Henry Karpenstein, 8o Ord St., San Francisco 17, Calif.

Albert Keiser, Lenoir Rhyne College, Hickory, North Carolina (College names)

Ralph W. Keller, Minnesota Editorial Assoc., 835 Palace Bldg., Minneapolis 1, Minn.

Allen B. Kellogg, 141 Loretta Drive, Indianapolis 3, Indiana

Arthur G. Kennedy, 1431 Arcadia Place, Palo Alto, Calif.

Hamill Kenny, P.O. Box 412, College Park, Md. (Indian place names of Maryland)

Marjorie M. Kimmerle, Room 121 Hellems, Univ. of Colorado, Boulder, Colo.

Watson Kirkconnell, Acadia University, Wolfville, Nova Scotia, Canada

Hans Krahe, Indogermanisches Seminar, Universität, Tübingen, Germany

Fritz L. Kramer, Allegheny College, Meadville, Penn. (Geographical names)

Hans Kurath, Angell Hall 5208, Univ. of Michigan, Ann Arbor, Michigan (Linguistic atlas)

Aneale L. Kushman, 2735 Taylor, Detroit 6, Michigan

Symington P. Landreth, I Pine Grove, Bristol, Penn.

Arthur D. Lane, 75 Newell Ave., Southbridge, Mass. (U.S. Personal and place names)

Don Lang, 831 S.W. 6th Ave., Portland 4, Oregon

John Leighly, 1435 Arch St., Berkeley 8, Calif.

Agnes C. Leland, 47 Avis Rd., Berkeley 7, Calif.

David Lindsay, Fair Elms, Berea, Ohio

Grant Loomis, 828 Coventry Rd., Berkeley 7 , Calif.

George D. Louderback, 107 Ardmore Rd., Berkeley 7, Calif. (Names in geology)

Hobart M. Lovett, 1177 Cragmont Ave., Berkeley 8, Calif.

Harold T. Lutz, 108 Dumbarton Rd., Baltimore 12, Md.

John Lyman, 7801 Gateway Blvd., Washington 28, D.C.

James G. W. Macclamroch, Jefferson Standard Bldg., Greensboron, N.C. (Gaelic, esp. Scot names)

W. L. McAtee, 3 Davie Circle, Chapel Hill, N.C. (Biological names, esp. plants and birds)

Eugene S. McCartney, 12 E. Mowry St., Chester, Penn. (Classical names)

John T. McCutcheon, Jr., "A Line of Type or Two," Chicago Tribune, Chicago 11, Ill.

John Francis McDermott, 6345 Westminster Place, St. Louis 5, Missouri

Marilyn E. MacGregor, 1915 Euclid Ave., Berkeley 8, Calif. 
James B. McMillan, Drawer 2877, University, Alabama

E. Wallace McMullen, 604 Belle View Blvd., Apt. A2, Alexandria, Va. (Topographic names, esp. Florida. Names in slang)

Alexander McQueen, 5222 N. Lakewood Ave., Chicago 40, Illinois

James M. McWilliams, 697 Arlington Ave., Berkeley 7, Calif.

Elsie Mag, G \& C Merriam Co., 47 Federal St., Springfield 2, Mass., (Lexicography)

Francis P. Magoun, Jr., 29 Reservoir St., Cambridge 38, Mass.

Kemp Malone, Dept. of English, Johns Hopkins University, Baltimore 18, Md. (Germanic and old English names)

Frederick F. Manfred, 6717 Auto Club Rd., Minneapolis 20, Minn. (Frisian names)

M. R. Manolatos, 5025 W. Monroe St., Chicago 44, Ill.

Carl H. Mapes, Rand McNally Co., Box 76oo, Chicago 8o, Ill.

Albert H. Marckwardt, University of Michigan, Ann Arbor, Michigan

Max Markrich, 248 Fifteenth Ave., San Francisco 18, Calif. (Semitic names)

Charles D. Matthews, House 1810, Aramco, Dharan, Saudi Arabia

Margot Mayo, 55o Riverside Dr., New York 27, N.Y.

Mamie Meredith, 116 Andrews Hall, Univ. of Nebraska, Lincoln 8, Nebr. (Geographical names and nicknames. Political terminology)

A. F. Michaelis, Standard Oil Co., 225 Bush St., Rm. 2174, San Francisco 20, Calif.

Guy C. Miller, 760 University Ave., Palo Alto, Calif. (California place names)

William E. Mockler, Dept. of English, West Virginia University, Morgantown, W.Va.

S. G. Morley, 3635 Etna St., Berkeley 4, Calif.

John G. Mutziger, 4711 Riverdale Road, Riverdale, Md. (Geographic names)

Delf Norona, 315 Seventh St., Moundsville, W.Va., (West Virginia and Ohio Valley names. Generics in colonial literature)

Maxwell Nurnberg, 520 Westminster Rd., Brooklyn 18, N.Y.

Clarence B. Odell, Denoyer-Geppert Co., 5235 Ravenswood Ave., Chicago 40, IIl.

Allan H. Orrick, Dept. of English, Johns Hopkins University, Baltimore 18, $\mathrm{Md}$.

Wm. D. Overman, 31 Mull Ave., Akron 3, Ohio (Ohio place names)

P. E. Palmer, Chairman, Canadian Board on Geographical Names, Ottawa, Canada

T. S. Palmer, 1939 Biltmore St., N.W., Washington, D.C. (Place Names of Death Valley)

T. M. Pearce, Univ. of New Mexico, Albuquerque, New Mexico (New Mexico place names)

Alfred Percy, Elon Rd., Madison Heights, Virginia (Central Virginia place and folk names)

Karl Peterson, 16o Felton Drive, Menlo Park, Calif. (Scandinavian names in lumbering)

Charles A. Philhower, 330 Mountain Ave., Westfield, New Jersey

Louise Pound, ${ }_{1632}$ L Street, Lincoln 8, Nebr.

Newbell N. Puckett, Western Reserve University, Cleveland 6, Ohio

Mrs. H. Pugh, 130 West Broad St., Burlington, New Jersey (Welch names)

Ernst Pulgram, Univ. of Michigan, Ann Arbor, Mich.

Thomas Pyles, 1618 N.W. 6th Ave., Gainesville, Fla. 
H. F. Raup, Dept. of Geography \& Geology, Kent State University, Kent, Ohio

Allen Walker Read, 39 Claremont Ave., New York 27, N.Y.

Click Relander, 1212 N. 32nd Ave., Yakima, Washington (Washington Indian names)

George Rentz, Box 1437, Arabian American Oil Co., Dhahran, Saudi Arabia

Norman L. Reynolds, Bear, Delaware

Philip M. Rhodes, \% Cameo Publications, P.O. Box 542, Tujunga, Calif. (Names of people and pets)

John S. Roberts, 469 3rd Street West, Sonoma, Calif.

R. D. Roberts, 312 Shearer Bldg., Bay City, Michigan (American surnames)

Karel Roelandts, Sergeysstraat 3, Herent, Belgium

Morris Rosenblum, 959 Carroll St., Brooklyn 25, N.Y.

Hubert A. Royster, 2318 Beechridge Road, Raleigh, N.C. (Medical nomenclature. Personal names).

J. B. Rudnyckyj, Dept. of Slavic Studies, Univ. of Manitoba, Winnipeg, Canada Sidney Rushford, Chaplain, St. Peter's Hospital, 380 Henry St., Brooklyn 2, N.Y.

William Saroyan, 24848 Malibu Road, Malibu, Calif. (Names in literature)

William M. Seaman, Dept. of Foreign Languages, Mich. State College, East Lansing, Mich.

Pauline A. Seeley, Denver Public Library, Denver 2, Colorado (Place name bibliography)

Alfred Senn, ${ }_{207}$ Cynwyd Rd., Bala-Cynwyd, Pa. (Baltic names)

George Pope Shannon, 4522 Drummond Ave., Chevy Chase 15, Md.

Esther K. Sheldon, 143-19 38th Ave., Flushing, N.Y.

Allan Morgan Standish, ${ }_{15} 15^{\circ}$ Via Colina, Los Gatos, Calif.

Taylor Starck, Harvard University, Cambridge 38 , Mass.

D. T. Starnes, Univ. of Texas, Austin 12, Texas.

W. A. Starr, 405 Montgomery St., San Francisco 4, Calif.

Vilhjalmar Stefansson, Dartmouth College Library, Hanover, New Hampshire

Jess Stein, Random House, 457 Madison Ave., New York 22, N.Y.

Mary Florence Steiner, ${ }_{25}$ Central Park Ave., Wilmette, Ill. (Spanish personal names)

Noel C. Stevenson, Yuba City, Calif.

George R. Stewart, 100 Codornices Rd., Berkeley, Calif.

Sterling A. Stoudemire, Univ. of North Carolina, Chapel Hill, North Carolina

O. Paul Straubinger, 4607 Oakwood Place, Riverside, Calif. (Names in proverbs, etc.)

Arval L. Streadbeck, A256 University of Utah, Salt Lake City 1, Utah

William Sunners, 1269 E. 49th Street, Brooklyn 34, N.Y.

E. G. Swem, 119 Chandler Court, Williamsburg, Virginia

John P. Sydoruk, 1607 N. Humboldt Blvd., Apt. 206, Chicago 47, Ill. (Slavic proper names)

Ashley F. Talbot, 317 William Street, East Orange, New Jersey (Place names in U.S. and in Oceania. Names on maps.)

Peter Tamony, 2876 24th St., San Francisco 10, Calif. (Folk names)

Edward Taube, Univ. of Wisconsin, 620 Lake Ave., Racine, Wis.

Gutierre Tibón, Paseo De La Reforma, Ciudad de Mexico, Mexico (IndoEuropean names)

Priscilla Tyler, 1495 Holmden Rd., South Euclid, Ohio

Harlan L. Umansky, Emerson High School, Union City, New Jersey 
Francis L. Utley, Ohio State University, Columbus 14, Ohio

Frank H. Waskey, Dillingham, Alaska (Geographic names. Onomatopoetic names in Eskimo)

R. Gordon Wasson, 23 Wall St., New York 8, N.Y.

Marie Waugh, 7 Beechwood Rd., Berona, N.J.

Robert H. Weidman, P.O. Box 306, Placerville, Calif. (English and German surnames)

Carl M. Weisman, 270 Van Siclen Ave., Brooklyn 7, N.Y. (Greater New York names)

Mrs. Carl Wesebaum, 15320 Waughan Ave., Detroit 23, Mich.

C. A. Weslager, 6o1 S. Maryland Ave., Wilmington, Delaware (Indian place names)

Robert C. West, Louisiana State University, Baton Rouge 3, La.

Louise B. Wheeler, P.O. Box 43, Davis, Calif.

B. J. Whiting, 3o Walker St., Cambridge 38, Mass.

H. J. Van De Wijer, E. Van Evenstraat 10, Louvain, Belgium

Viola Willeke, 540 N. Glemdale, Kenton, Ohio

George P. Wilson, 1813 Rolling Road, Greensboro, N.C.

Ralph Emerson Woods, 106 Millar Ave., San Jose 27, Calif. (Place names. Names in slang)

H. B. Woolf, Louisiana State Univ., Baton Rouge 3, Louisiana

A. J. Wraight, 18 gth St., N.E., Washington 2, D.C. (Coast and Geodetic Survey)

Gertrude M. Zimmer, 221 Patterson St., Farmington, Missouri 